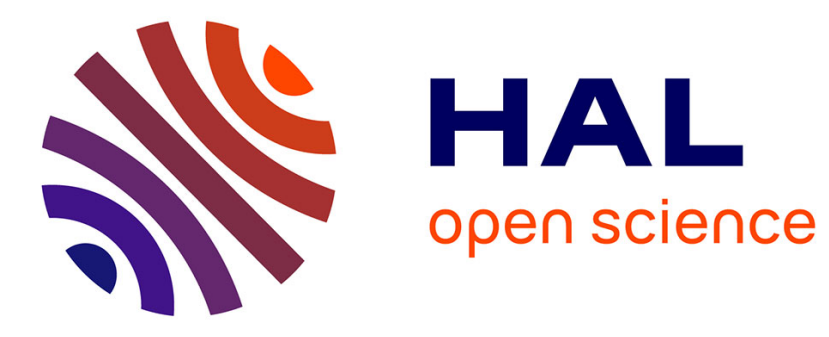

\title{
Les composites multifilamentaires (condensé)
}

G. Bronca, J.P. Pouillange, G. Prost

\section{To cite this version:}

G. Bronca, J.P. Pouillange, G. Prost. Les composites multifilamentaires (condensé). Revue de Physique Appliquée, 1971, 6 (4), pp.527-530. 10.1051/rphysap:0197100604052700 . jpa-00243582

\section{HAL Id: jpa-00243582 https://hal.science/jpa-00243582}

Submitted on 1 Jan 1971

HAL is a multi-disciplinary open access archive for the deposit and dissemination of scientific research documents, whether they are published or not. The documents may come from teaching and research institutions in France or abroad, or from public or private research centers.
L'archive ouverte pluridisciplinaire HAL, est destinée au dépôt et à la diffusion de documents scientifiques de niveau recherche, publiés ou non, émanant des établissements d'enseignement et de recherche français ou étrangers, des laboratoires publics ou privés. 


\title{
LES COMPOSITES MULTIFILAMENTAIRES (condensé)
}

\author{
G. BRONCA. J.P. POUILLANGE et G. PROST \\ Département Saturne G. A.T.S. \\ Commissariat à l'Energie Atomique, Saclay.
}

\begin{abstract}
Résumé. - Le comportement électromagnétique des composites multifilamentaires est compliqué par les raisons suivantes :

- Les filaments supraconducteurs n'étant pas isolés, des boucles de courant de circulation se referment entre filaments à travers le métal de la matrice à l'intérieur d'un demi-pas de torsade. Les pertes dues à ces courants sont évaluées dans le cas où le composite ne transporte pas de courant. - L'opération de torsadage ne constitue pas une transposition parfaite des filaments par rapport au champ magnétique propre au composite. Les effets du champ propre sur la distribution du courant dans le composite et les pertes électriques sont indiqués. tique.

- Par suite de leur torsadage les filaments ne sont pas partout orthogonaux au champ magné-

Les résultats expérimentaux concernant la dégradation et les pertes de petites bobines réalisées à l'aide de plusieurs composites multifilamentaires sont résumés.
\end{abstract}

\begin{abstract}
The electro-magnetic behaviour of the multifilamentary composites is complicated by the following causes :

- The superconducting filaments are not insulated thus circulating current loops are closed in half a twist pitch through superconducting filaments and matrix metal. The losses due to these currents are estimated in the case where there is no transport current.

- The twist operation is not a perfect transposition of the filaments in the composite selffield. Self-field effects on the current distribution in the composite and on the losses are indicated.

- Due to the twist the filaments are not everywhere perpendicular to the magnetic field.

Experimental results concerning the degradation and the losses of small coils made from several multifilamentary composites are reviewed.
\end{abstract}

I. Introduction. - On sait que les pertes d'un filament supraconducteur dur de deuxième espèce plongé dans un champ magnétique extérieur variable normal à son axe sont proportionnelles à son diamètre. Elles sont typiquement de $6 \times 10^{-2}$ joule par centimètre cube de matériau et par cycle pour un filament de $\mathrm{Nb}$-Ti de diamètre $10 \mu$ exposé à un champ d'induction magnétique pulsé, dont la valeur de crête est 6 teslas (onde triangulaire, ou trapézoïdale d'induction variant périodiquement de 0 à 6 teslas).

L'application de ces supraconducteurs à la réalisation d'aimants de synchrotron serait intéressante dans la mesure où le niveau des pertes par unité unité de volume de matériau supraconducteur et par cycle correspondrait à celui de filaments uniques de diamètre $4 \mu$.

Il n'est pas question de produire individuellement des filaments de cette finesse. On en coétire en fait, plusieurs centaines simultanément dans une matrice de métal normal (composite multifilamentaire). Si, à la suite de cette opération, on alimentait en courant pulsé le conducteur ainsi obtenu, on obtiendrait des pertes par cycle supérieures à celles d'un filament unique de section égale à la somme des sections des filaments contenus dans le composite. Cela provient des courants de circulations qui existent entre ces filaments parallèles alimentés en parallèle, d'où l'idée de les transposer. Le seul mode de transposition possible, dans ce cas, consiste à torsader les filaments dans la matrice par torsion permanente du composite.

Ce torsadage améliore considérablement le comportement du composite, mais il présente toutefois des imperfections résiduelles que nous nous proposons d'analyser :

1) Les filaments supraconducteurs n'étant pas isolés de la matrice, il se boucle encore entre filaments à l'intérieur d'un demi-pas de torsade des courants de circulation à travers la matrice de métal normal.

2) La transposition par torsadage est parfaite par rapport au champ extérieur au composite, mais incomplète par rapport au champ magnétique engendré par le courant transporté par le composite lui-même, dit champ propre.

3) Pour réduire le niveau des pertes entraînées par la première imperfection, on est amené à opter pour des pas de torsade courts et, de ce fait, si le champ extérieur est normal au composite, il ne l'est plus que localement aux filaments eux-mêmes.

Notre propos est d'étudier l'influence de ces trois imperfections sur les pertes et la stabilité du composite, 
puis de résumer les résultats expérimentaux obtenus sur des composites torsadés dont les filaments ont un diamètre voisin de $10 \mu$.

Ce résumé sera très bref et incomplet; le lecteur intéressé est prié de se reporter au rapport GATS/71-24 $\mathrm{du}$ 18.3.1971 (Les composites multifilamentaires).

II. Aspect théorique. - II.1 Courants DE CIRCULATION INTÉRIEURS AU DEMI-PAS DE TORSADE. - On suppose la densité critique $J_{\mathrm{c}}$ des filaments et la résistivité $\rho$ de la matrice indépendantes de l'induction $B$. Si $|\mathrm{d} B / \mathrm{d} t|$ est suffisamment faible pour que le courant de circulation intéressant un filament périphérique soit petit relativement au courant critique de ce filament (cas pratique), il n'aggrave guère les pertes dans le supraconducteur, mais provoque dans le métal normal des pertes $A_{\mathrm{N}}$ par cycle et unité de volume.

$$
\begin{aligned}
A_{\mathrm{N}}=\frac{1}{\rho} \frac{P^{2}}{4 \pi^{2}}\left[1+\frac{\sqrt{3} a}{2 d}\right]^{2} & \times \\
\times & {\left[1+\frac{1}{4} \operatorname{tg}^{2} \varphi_{(R)}\right]\left|\frac{\mathrm{d} B}{\mathrm{~d} t}\right| B_{\max } }
\end{aligned}
$$

où $P$ est le pas de torsade du composite ;

$\varphi_{(R)}$ est l'angle que font les filaments périphériques avec l'axe du composite ;

$\rho$ la résistivité de la matrice ;

$B_{\max }$ l'induction de crête.

$a$ et $d$ sont précisés sur la coupe orthogonale à l'axe du composite (voir Fig. 1).

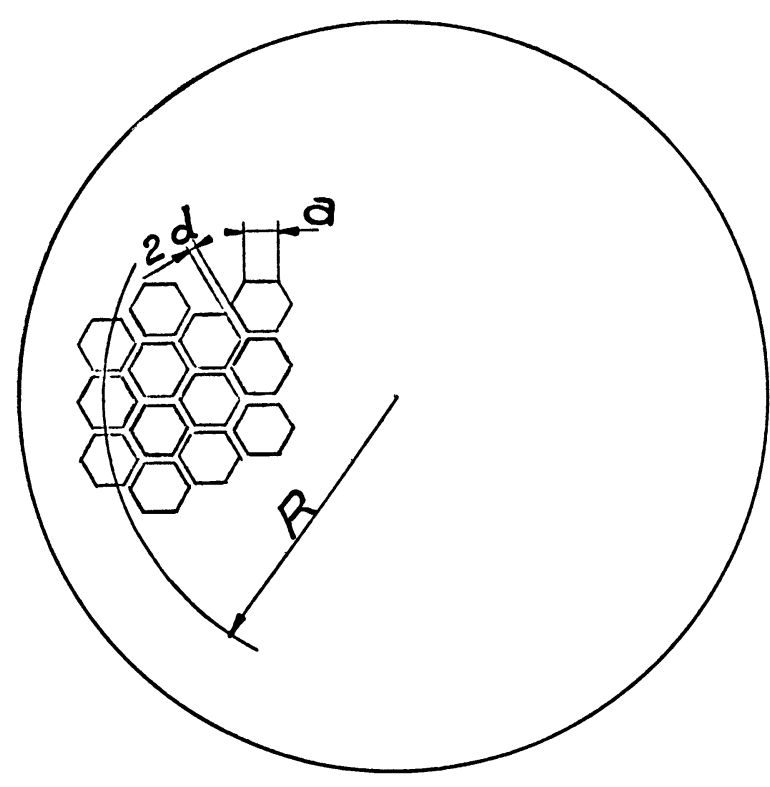

Fig. 1. - Coupe du composite normale à son axe.

Application numérique. - Caractéristiques du composite : $R=185 \mu, P=2 \mathrm{~mm}$ (d'où $\varphi_{(R)} \# \pi / 6$ ), $a=5,18 \mu, \tau=0,65$ (taux d'occupation de la zone filamentaire par le supraconducteur), $\rho=10^{-9} \Omega . \mathrm{m}$ (bon cuivre + effet dimensionnel) $J_{\mathrm{c}}=2 \times 10^{9} \mathrm{~A} / \mathrm{m}^{2}$.
$\mathrm{Si}|\mathrm{d} B / \mathrm{d} t|=4 \mathrm{~T} / \mathrm{s}$ et $B_{\max }=6$ teslas, on en déduit que les pertes dans le cuivre s'élèvent à $76 \%$ des pertes dans le supraconducteur.

En ce qui concerne la stabilité du composite, tant que la somme du courant de transport et du courant de circulation dans les filaments périphériques est inférieure au courant critique de ces filaments, il existe une surface de champ électrique nulle dans chaque filament; du point de vue de la stabilité, chacun d'eux se comporte comme s'il était seul et s'ils sont suffisamment fins, le composite est adiabatiquement stable. Par contre, à l'approche du courant de transport critique, il n'en est plus ainsi et pour de gros composites, on peut être amené à compter sur la stabilité dynamique.

II. 2 EFFET DU CHAMP PROPRE. - Si le champ extérieur est très grand relativement au champ propre du composite, la transposition parfaite par rapport au champ extérieur l'emporte et la distribution du courant entre les différents filaments est régulière.

Par contre, si le champ extérieur est faible (zone bas champ d'une bobine), le courant varie beaucoup plus vite dans la couche périphérique que dans les couches intérieures, et lorsqu'elle transporte son courant critique, c'est la couche suivante qui joue le rôle de couche périphérique, etc...

Le cas le plus général est intermédiaire entre les deux précédents.

On peut voir que l'effet du champ propre sur les pertes du composite n'est pas considérable.

En ce qui concerne la stabilité, elle est assurée par la finesse des filaments tant que les couches externes ne transportent pas leur courant critique. Dans le cas contraire, on voit que le risque d'instabilités est plus marqué, lorsque le composite, ayant été refroidi en l'absence de champ magnétique, est soumis à la première montée du courant. Nous avons établi un critère de stabilité adiabatique qui fixe le rayon maximum du composite. Ce critère ne semble pas draconien et on peut encore l'adoucir en faisant appel à la stabilité dynamique du composite.

II. 3 NON-ORTHOGONALITÉ DU CHAMP ET DES FILAMENTS. - Nous nous proposons de définir expérimentalement l'effet de l'angle, que fait avec le champ magnétique la direction des lignes de courant, sur la valeur de la densité de courant critique dans le cas de filaments fins. Une fois cette loi obtenue, nous serons à même d'étudier la distribution du champ électrique dans un filament supraconducteur soumis à un champ magnétique non orthogonal à son axe et d'en déduire les pertes en régime variable.

Une augmentation des pertes due à cette non-orthogonalité est prévisible. Les pertes resteront toutefois proportionnelles au diamètre du filament.

III. Résultats expérimentaux. - Nos essais ont porté sur trois conducteurs IMI et un conducteur Supercon dont les caractéristiques sont indiquées dans le tableau suivant: 


\section{Nombre de} composites

IMI TC

IMI $1045 / 33$

IMI $1000 / 44$

Supercon

A l'aide de ces câbles, nous avons réalisé différentes bobines dont les dimensions étaient typiquement: diamètre intérieur : $2 \mathrm{~cm}$, diamètre extérieur : $8 \mathrm{~cm}$, longueur : $9 \mathrm{~cm}$. Ces bobines étaient refroidies soit par un système de canaux verticaux de profondeur variable, soit par l'intermédiaire de nappes de fils de cuivre isolés (afin de réduire les pertes Foucault en régime variable), disposées entre couches et débouchant dans l'hélium aux deux extrémités de la bobine. Ces dernières bobines étaient, de plus, imprégnées de graisse.

ETUDE DE LA DÉGRADATION. (Rapport du courant de blocage mesuré sur bobine au courant prévu à partir de la loi de courant critique mesuré sur échantillon court en fonction de l'induction.)

- Pas d'accommodation à l'exception des bobines réalisées à l'aide du câble Supercon dont le comportement est plus singulier (diffusion cuivre- $\mathrm{Nb}$ ?). rant.

- Pas de dégradation en montée très lente du cou-

- Lorsque la bobine est alimentée en courant pulsé, la dégradation du courant de blocage est d'autant plus importante que la fréquence est plus élevée (voir courbe 2). Cette dégradation progressive peut s'expliquer par le fait que la puissance de pertes, fonction croissante de la fréquence, entraîne une élévation de la température de la bobine d'où une diminution du courant critique du câble.

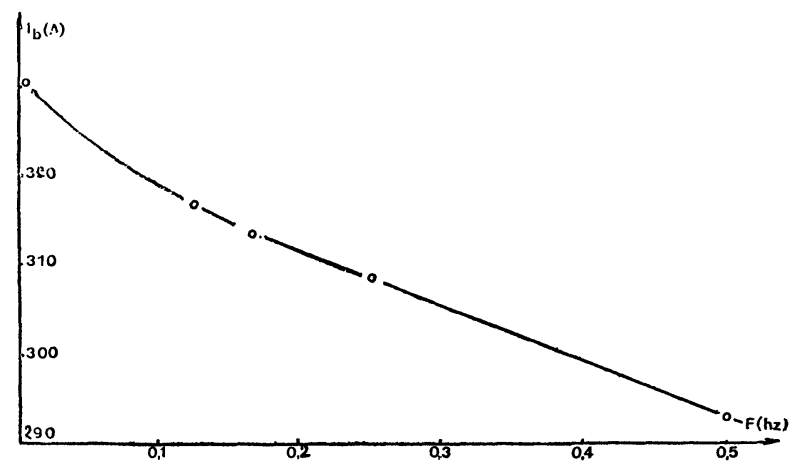

Fig. 2. - Dégradation de $I_{b}$ en fonction de F câble IMI $1000 / 44$.

ETUDE DES PERTES. - La bobine alimentée en courant pulsé engendre son propre champ ; ses pertes sont mesurées par méthode électrique avec une précision meilleure que $10 \%$.

On peut faire les constatations suivantes :

- Les courbes 3 qui portent sur l'ensemble des bobines testées dans le laboratoire montrent que le quotient des pertes par cycle et par unité de volume de $\varnothing$

lament $(\mu)$

Nature matrice

métal norm. supra.

Pas de torsade $(\mathrm{mm})$

10
6,6
10
7,5

$\overline{39}$
1,39
1
1

2

2

supraconducteur par la densité critique du matériau varie proportionnellement avec la taille des filaments comme le prévoit la théorie. Cette proportionnalité est en défaut pour les filaments de fort diamètre. Cet écart s'explique par le fait que la proportionnalité n'est vraie que si l'on néglige les périodes de pénétration du supraconducteur par le flux. Dans le cas de gros filaments, cette période de pénétration n'est plus négligeable devant le temps d'excursion de l'induction magnétique.

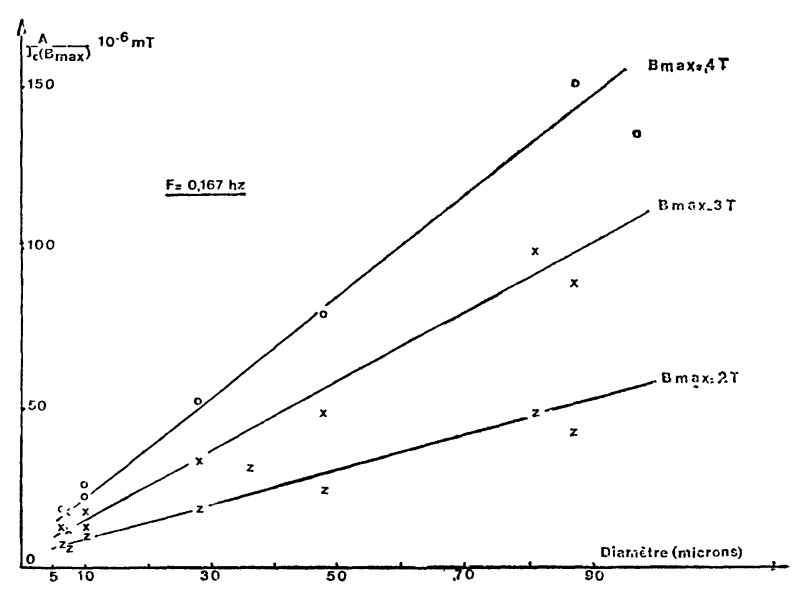

FIG. 3.

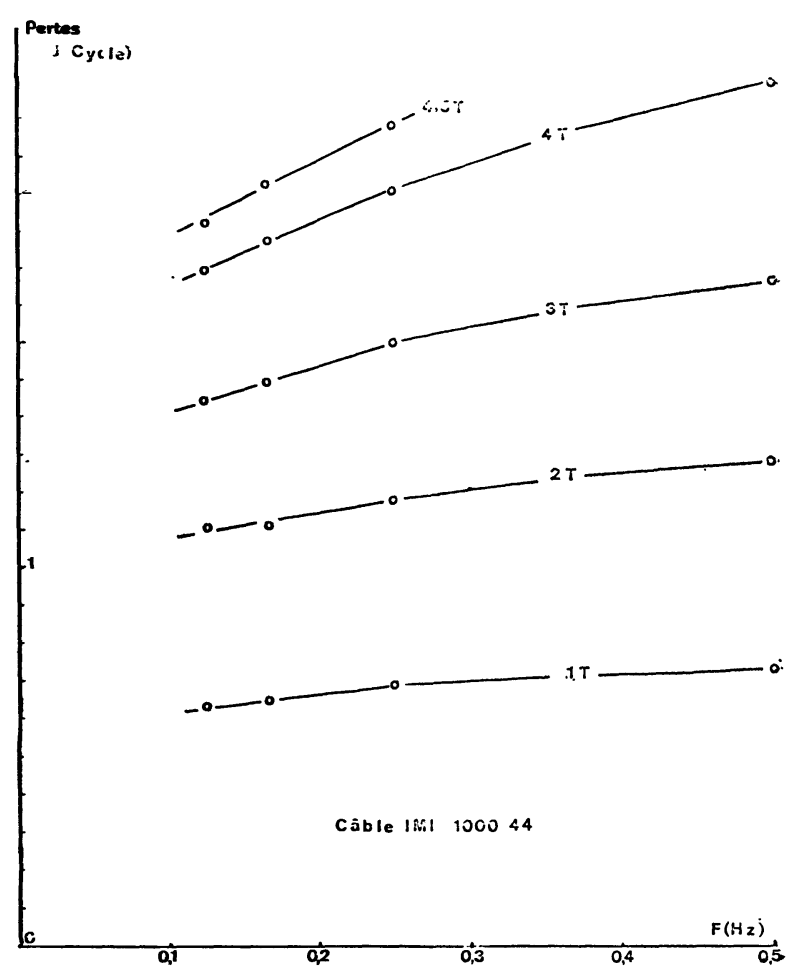

FIG. 4. 
- Les courbes 4 montrent la linéarité des pertes en fonction de la fréquence dans le domaine des basses fréquences.

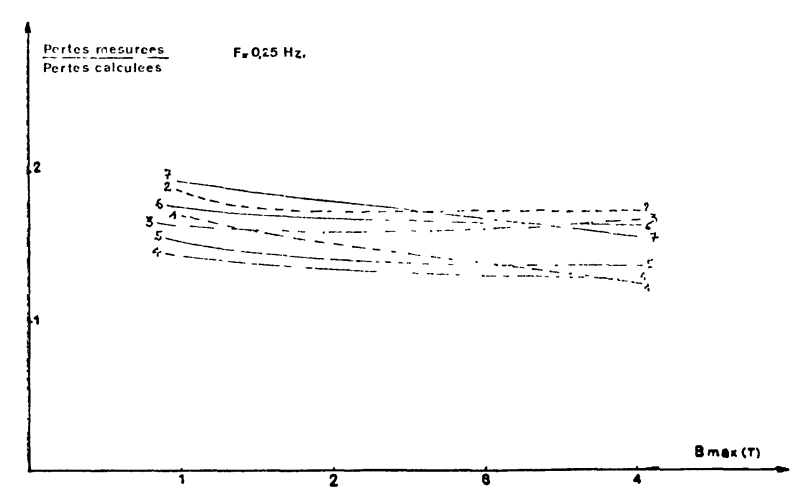

Fig. 5. - $1^{\circ}$ IMI $1000 / 44$ canaux. $2^{\circ}$ IMI TC $1045 / 50$. $3^{\circ}$ Supercon canaux $0.5 \mathrm{~mm}$. $4^{\circ}$ Supercon canaux $1 \mathrm{~mm}$. $5^{\circ}$ Supercon drains. $6^{\circ}$ IMI $1045 / 33$ canaux. $7^{\circ}$ IMI $1045 / 33$ drains.
- Enfin, nous avons calculé le rapport des pertes mesurées aux pertes calculées à partir d'un programme qui remplace le composite par un ensemble idéal de filaments isolés et transposés. On peut voir que ce rapport reste inférieur à deux sur les courbes 5 où il est exprimé pour les différentes bobines en fonction du champ de crête $B_{\max }$, la fréquence de pulsation étant de $0,25 \mathrm{~Hz}$. L'écart de ces pertes représente la contribution des termes additionnels indiqués dans le paragraphe II ainsi qu'éventuellement les pertes liées à des déplacements de spires.

IV. Conclusion. - Les résultats expérimentaux sont en bon accord avec la théorie. Si, toutes choses égales par ailleurs, on diminue encore la taille des filaments, il sera bon, toutefois, de choisir dans la zone filamentaire une matrice plus résistive afin de réduire dans les mêmes proportions que les pertes dégagées dans le supraconducteur, les pertes liées aux courants de circulation dans le métal normal. 